\title{
In Situ and Cryo (S)TEM Imaging of Internal Microgel Architectures
}

Tobias Caumanns ${ }^{1}$, Arjan Gelissen ${ }^{2}$, Alexander Oppermann ${ }^{2}$, Pascal Hebbeker ${ }^{2}$, Rahul Tiwari ${ }^{3}$, Sarah Turnhoff ${ }^{2}$, Dominik Wöll ${ }^{2}$, Andreas Walther ${ }^{3}$, Joachim Mayer ${ }^{1}$ and Walter Richtering ${ }^{2}$

1. Central Facility for Electron Microscopy, RWTH Aachen University, Aachen, GERMANY

2. Institute of Physical Chemistry, RWTH Aachen University, Aachen, GERMANY

3. Leibniz-Institute for Interactive Materials, RWTH Aachen University, Aachen, GERMANY

Microgels are responsive polymer materials and structures, which can be manipulated in size by external parameters, in this case by temperature variation. They are dispersed in an aqueous medium and establish an ideal basis to develop smart bio-inspired materials. The big advantage of microgels in contrast to rigid colloidal particles, are switchable properties of form and function, which are very useful in e.g. biological sciences and medical applications.

To analyse the spatial distribution of different compartments and design novel materials, it is important to directly visualize the internal structure of materials. Microgels can be prepared with various morphologies and functions in different compartments. Careful analysis of the correlation between architecture and function requires powerful methods to visualize inner structure and compartmentalization in the nanometer range.

This work shows the direct visualization of different compartments within microgels via in situ liquid cell and cryo transmission electron microscopy. Different compartments of the microgel particles are labelled by heavy metal nanoparticles to increase the contrast in the (S)TEM. The challenge to determine radial distributions of nanoparticles from $2 \mathrm{D}$ projections is addressed. To validate the (reversible) shrinking/swelling behaviour of the microgel the liquid sample is heated up and the whole process is recorded with the HAADF detector of the TEM.

Spherical core-shell microgels were obtained by precipitation polymerization. Core and shell were step by step synthesized by the seed and feed method. Both, core and shell, have oppositely charged copolymers to create a two compartment microgel system alternately stained with gold, magnetite or silver nanoparticles $[1,2,3]$.

For in situ liquid cell experiments, a thin layer of liquid was embedded between two hermetically sealed, electron transparent $\mathrm{Si}_{3} \mathrm{~N}_{4}$-windows. For the heating experiments a special $\mathrm{Si}_{3} \mathrm{~N}_{4}$-window with two electrical contacts to apply a heating current was placed above the liquid layer. A control box carried out continuous measurements of the resistance to calculate the current temperature. The used holder is an in situ liquid cell holder and the $\mathrm{Si}_{3} \mathrm{~N}_{4}$-windows are manufactured by Hummingbird Company and the microscope is a Zeiss Libra 200FE with an acceleration voltage of $200 \mathrm{kV}$. The resolution is mainly limited by the thickness of the liquid $[4,5]$.

\section{References:}

[1] Y Lu et al, J Mater Chem 19 (2009), pp. 3955-3961.

[2] O Mergel et al, J Phys Chem C 118 (2014), pp. 26199-26211.

[3] J Kleinen, A Klee and W Richtering, Langmuir 26 (2010), pp. 11258-11265. 
[4] N de Jonge, F M Ross, Nat Nanotechnol 6 (2011), pp. 695-704.

[5] A Gelissen et al, Nano Lett. 2016 16, pp. 7295-730.

[6] The authors kindly acknowledge the financial support by the DFG through the SFB 985.

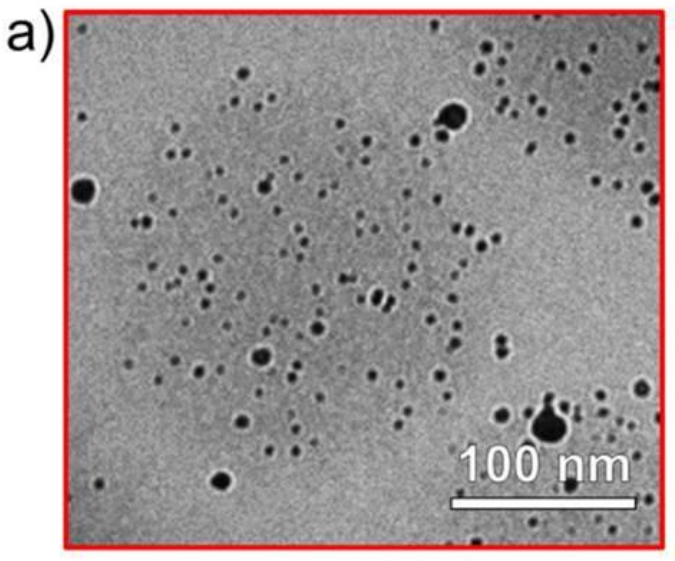

cryo TEM

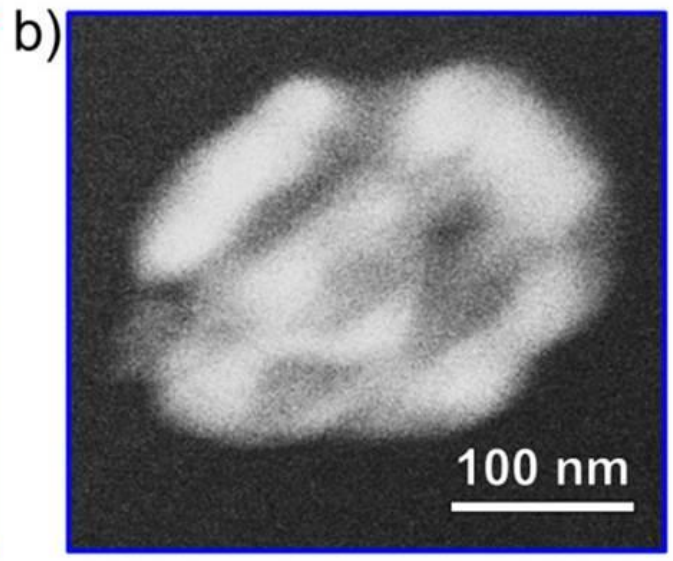

in situ TEM

Figure 1. Comparison of cryo- and in situ TEM (a) BF (Bright field) cryo-TEM image of a microgel with core-shell architecture stained with Ag-NPs. (b) In situ high angle annular dark field scanning TEM (HAADF STEM) image of the same microgel [5], with permission from the American Chemical Society.

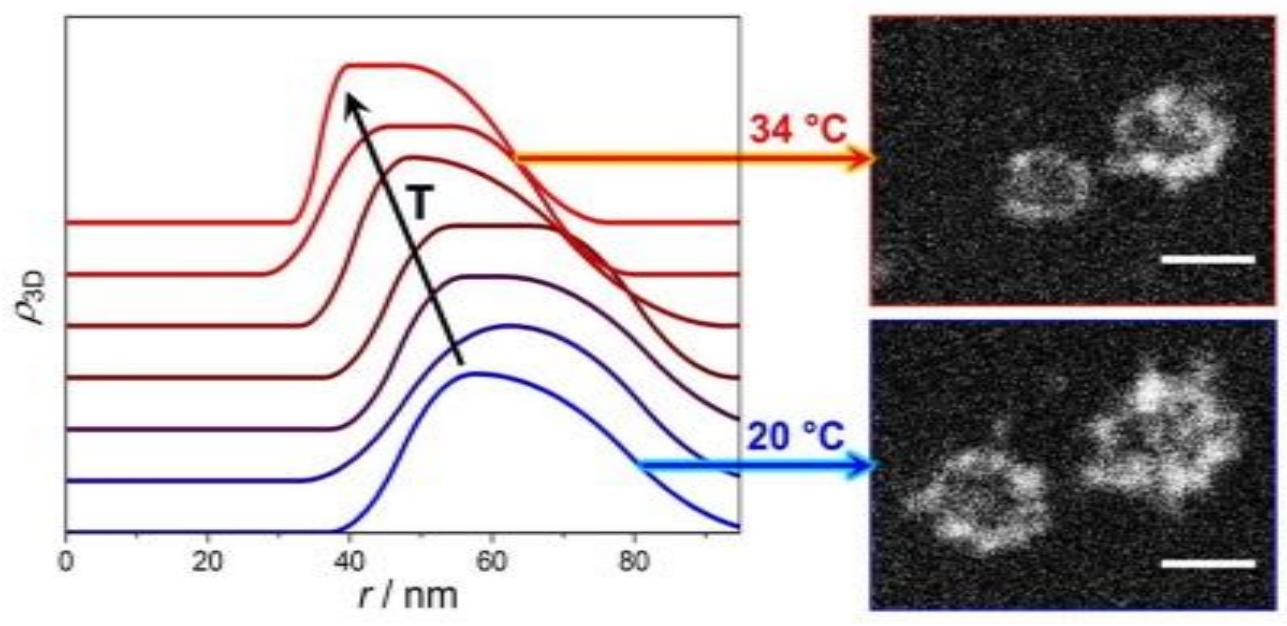

Figure 2. Temperature-dependent collapse of a PNIPAM-based microgel stained with AgNPs [5], with permission from the American Chemical Society. 